\section{ORIGINAL RESEARCH}

S. Liu

J.H. Jung

S.-M. Kim

H.-K. Lim

H.-j. Kwon

J.K. Kim

J.S. Kim

D.C. Suh

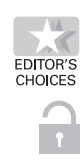

\title{
Simultaneous Bilateral Carotid Stenting in High-Risk Patients
}

BACKGROUND AND PURPOSE: The safety and efficacy of SBCAS have not been evaluated in detail. The purpose of our study was to evaluate the outcome after SBCAS in high-risk patients compared with unilateral stent placement.

MATERIALS AND METHODS: Between March 2002 and October 2008, a total of 205 consecutive high-risk patients underwent CAS at our institution. Of these patients, 30 (14.6\%) underwent SBCAS $(n=24)$ and staged SBCAS $(n=6)$. Patients who underwent unilateral CAS ( $n=175)$ during the same period served as controls. The stroke risk factors, procedural results, and outcome at 30 days and 6 months, as well as the restenosis rate at 6 months, were compared by using either the $\chi^{2}$ test or the Kruskal-Wallis equality-of-populations rank test.

RESULTS: Our data revealed no significant differences in the stroke risk factors between the SBCAS and the control group. HPS occurred more commonly in SBCAS (ie, 16.7\%, 4/24) compared with $2.9 \%$ (5/175) in the control group $(P=.014)$. However, there was no statistical significance between 2 groups in the event rate of stroke (minor and/or major stroke), death, or restenosis at 6 months.

CONCLUSIONS: There was no significant difference in outcome at 6 months following stent placement between SBCAS and unilateral CAS in the high-risk patient group, even though HPS occurred more commonly after SBCAS.

ABBREVIATIONS: $\mathrm{BP}=$ blood pressure; $\mathrm{CAS}=$ carotid artery stent placement; $\mathrm{CEA}=$ carotid endarterectomy; CCS = Canadian Cardiovascular Society; FEV = forced expiratory volume; $\mathrm{HD}=$ hemodynamic depression; HPS = hyperperfusion syndrome; mRS = modified Rankin scale; NASCET $=$ North American Symptomatic Carotid Endarterectomy Trial; NIHSS $=$ National Institutes of Health Stroke Scale; NYHA = New York Heart Association; SBCAS = simultaneous bilateral carotid artery stent placement; TIA = transient ischemic attack

$\mathbf{S}^{\mathrm{n}}$ roke is the leading cause of serious long-term disability as well as a growing burden for governments and patients. Approximately $50 \%$ of strokes occur in the carotid arteries. ${ }^{1,2}$ Despite the controversies regarding the optimal therapy for carotid artery stenosis, ${ }^{3}$ CAS by using a distal protection device is preferable to CEA for high-risk patients. ${ }^{4,5}$

Since Mathur et $\mathrm{al}^{6}$ first reported the feasibility of staged CAS for bilateral carotid stenosis, some small series have been published in an attempt to promote staged or simultaneous revascularization. ${ }^{7-16}$ However, the results have been equivocal, and there is an obvious need for larger trials to firmly establish the superiority of the simultaneous or the staged approach. Nonetheless, patients with bilateral carotid artery disease have been excluded from most prospective trials due to concerns regarding possible complications and/or poor outcomes. $^{17}$

\section{Received August 4, 2009; accepted after revision October 24.}

From the Department of Radiology and Research Institute of Radiology (S.L., J.H.J., S.-M.K., H.-K.L., H.-j.K., D.C.S.) and Department of Neurology (J.S.K.), University of Ulsan, College of Medicine, Asan Medical Center, Seoul, Korea; Department of Radiology (S.L.), First Affiliated Hospital of Nanjing Medical University, Nanjing, China; and Department of Radiology (J.K.K.), Seoul Veterans Hospital, Seoul, Korea.

This study was supported by a grant from the Asan Institute for Life Sciences (2009-143), Seoul, Korea.

Please address correspondence to Dae Chul Suh, MD, Department of Radiology, Asan Medical Center, University of Ulsan, College of Medicine, 388-1 Pungnap2-dong, Songpagu, Seoul, 138-736, Korea; e-mail: dcsuh@amc.seoul.kr

Indicates open access to non-subscribers at www.ajnr.org

DOI 10.3174/ajnr.A1970
Bilateral carotid severe stenosis, presumed to be 1 of the high risk factors in CEA, is generally treated by a staged stentplacement procedure..$^{7-9,17}$ On the other hand, despite the additional medical cost and physical burden to the patient of using the staged procedure, simultaneous SBCAS is not usually recommended because of the risk of cerebral HPS and HD, such as severe bradycardia or hypotension. ${ }^{17,18}$

Some reports supporting SBCAS have demonstrated that it is feasible and has theoretic advantages over staged stent placement, including potentially minimizing both hospitalization and medical costs. ${ }^{10-15}$ However, the literature reporting SBCAS is mostly small series, and the available data regarding SBCAS are limited. In this study, we retrospectively analyzed the short-term safety and efficacy of SBCAS compared with control group results by using the single CAS procedure.

\section{Materials and Methods}

Among the 205 consecutive surgically high-risk patients who underwent CAS in our prospectively collected neurointerventional data base between March 2002 and October 2008, 30 patients (14.6\%) underwent SBCAS due to atherosclerotic carotid stenosis. We included these patients with symptomatic $>50 \%$ or asymptomatic $>80 \%$ stenosis on the basis of angiography by using the NASCET method. ${ }^{19}$ All patients were at high risk for CEA or had medical problems (Table 1). We excluded all patients who underwent stent placement during revascularization as part of their acute stroke management or who had a dissection or a nonatheromatous vascular lesion such as Takayasu arteritis.

The patient population with bilateral carotid bulb lesions $(n=30)$ included SBCAS $(n=24)$ and staged SBCAS $(n=6)$. Patients who 


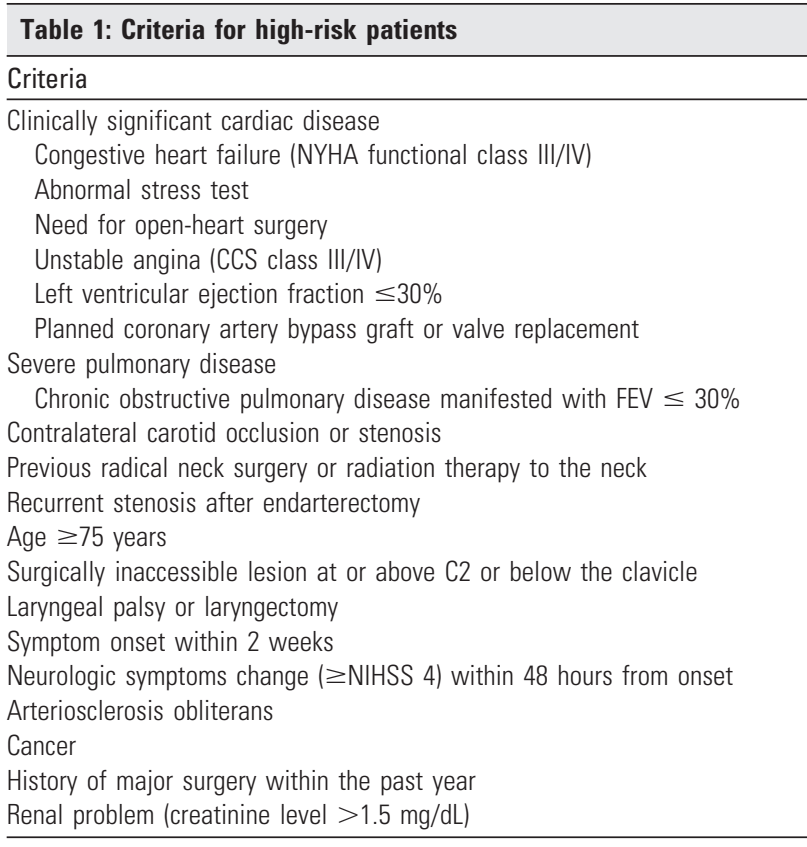

underwent unilateral CAS ( $n=175)$ served as controls. Our institutional review board approved this retrospective study, and all patients gave written informed consent for their treatment. A complete history was taken for each patient, and a neurologic examination was performed before and after the stent placement by independent neurologists who were not involved in the interventional procedure.

Imaging modalities before endovascular treatment included color duplex sonography of the carotid arteries and CT angiography or MR angiography of the aortic arch anatomy and intracranial vessels. Brain imaging was routinely performed by using diffusion-weighted MR imaging or MR imaging before the procedure.

Diffusion-weighted MR images obtained in 122 patients with unilateral lesions revealed new ischemic lesions in 84 patients-that is, borderzone $(n=54)$, wedge-shaped pial $(n=22)$, scattered superficial cortical $(n=4)$, basal ganglia $(n=3)$, and deep perforator $(n=1)$ lesion patterns of acute ischemic change. Diffusion-weighted MR images obtained in 22 patients with bilateral lesions revealed new ischemic lesions in 16 patients-that is, borderzone $(n=8)$, wedgeshaped pial $(n=5)$, and scattered superficial cortical $(n=3)$ lesion patterns of acute ischemic change. ${ }^{20}$

Nineteen (63\%) among the 30 patients with bilateral lesions had 1 symptomatic side based on the presenting symptoms and/or lesions seen on MR imaging. Two patients were regarded as having bilateral symptoms: One presented with symptoms related to the contralateral side within a 1-week interval, and the other presented with bilateral acute ischemic lesions on both sides of the middle cerebral artery territories on diffusion-weighted imaging. Five patients presented with either severe dizziness $(n=4)$ or cognitive dysfunction $(n=1)$, which were regarded as symptomatic but difficult to lateralize. Four asymptomatic patients were seen to have bilateral carotid stenosis noted during their work-up for carotid artery bypass surgery $(n=3)$ or a congestive heart failure $(n=1)$.

Dual antiplatelet medication (100 mg of aspirin and $75 \mathrm{mg}$ orally of clopidogrel) was started at least 3 days before the stent-placement procedure. If patients were not already taking these medications, 200 $\mathrm{mg}$ of aspirin and a loading dose of $300 \mathrm{mg}$ of clopidogrel were given to each patient before the procedure. We then continued them on 100 mg of aspirin once daily as a permanent medication. In addition, 75 mg of clopidogrel was given once daily for at least 3 months after the procedure.

\section{Techniques and Procedures}

All procedures were performed under monitored anesthesia care by an anesthesiologist, and there was continuous monitoring of intrarterial pressure, electrocardiography, and neurologic status throughout the procedure. Via a femoral route, a $6 \mathrm{~F}$ sheath or an $8 \mathrm{~F}$ guiding catheter was introduced below the common carotid artery bifurcation, and the sidearm was continuously flushed with pressurized heparinized normal saline. Systemic heparinization was given to achieve the activated clotting time of approximately 250 seconds. Baseline angiography was performed, and the lumen diameters of the stenotic lesions as well as of the adjacent arterial segments were measured by using the NASCET method ${ }^{19}$; the intracranial collaterals were also evaluated. A distal embolic protection device was used in 19 of the 24 patients $(79 \%)$.

After undersized balloon predilation was performed, a self-expandable stent was inserted into the stenotic portion of the carotid bulb. Atropine $(0.5 \mathrm{mg}$ ) was given intravenously to these patients as is required during balloon angioplasty and stent placement. After the balloon catheter was removed and the stent was deployed, we performed postdilation if there was residual stenosis of $>30 \%$. The final angiogram was obtained to evaluate the carotid artery and the intracranial vessels. In patients with bilateral carotid stenosis, the dominant lesion, which was defined as either the symptomatic side or the asymptomatic side with greater reduction in vessel diameter according to the imaging studies, was treated first. We used a protection device on both sides. After stent placement, all patients were transferred to the neurointensive care unit for continuous 24-hour observation. The BP was carefully monitored and controlled at $<120 / 80-$ 130/90 mm Hg according to each patient's baseline BP.

Twenty-four patients (11.7\%) in our series underwent SBCAS. Six patients underwent the procedure in a staged manner (2.9\%). Among the 175 control group patients, a distal embolic protection device was used in $131(74.9 \%)$ during their treatment. A total of 155 protection devices $(75.6 \%)$ were used during the stent-placement procedures: 124 FilterWires (Boston Scientific, Natick, Massachusetts), 15 Emboshields (Abbott, Chicago, Illinois), 13 Spiders (ev3, Plymouth, Minnesota), and 3 GuardWires (Medtronic Vascular, Minneapolis, Minnesota). The reasons for not using them included a tortuous common carotid artery, a narrow culprit lesion that did not allow passage of the device, or a tortuous distal internal carotid artery deemed unsuitable for distal placement of the device.

A total of 235 stents were inserted into 205 patients, including 112 (48\%) Wallstents (Boston Scientific), 97 (41\%) Precise stents (Cordis, Warren, New Jersey), and 26 (11\%) Zilver stents (William Cook Europe, Bjaeverskov, Denmark).

\section{Outcome Evaluations and Follow-Up}

Primary procedural success was defined as $<30 \%$ residual stenosis following stent placement. HPS was diagnosed as the occurrence of ipsilateral (to the treated artery) throbbing headache with or without nausea, vomiting, or ipsilateral focal seizures, or the presence a focal neurologic deficit without radiographic evidence of infarction. ${ }^{21}$ Perfusion-weighted MR images, CT perfusion, or single-photon emission tomography was performed for these patients with suspected HPS following the procedure. Any symptomatic or asymptomatic hypotension (systolic BP $<90 \mathrm{~mm} \mathrm{Hg}$ or bradycardia, ie, heart rate 
Table 2: Distribution of risk factors in patients with carotid stenting

\begin{tabular}{lcccc}
\hline & \multicolumn{2}{c}{ Bilateral Group } & Control Group \\
\cline { 2 - 4 } Risk factors & $\begin{array}{c}\text { Simultaneous } \\
(n=24)\end{array}$ & $\begin{array}{c}\text { Staged } \\
(n=6)\end{array}$ & $\begin{array}{c}\text { Unilateral } \\
(n=175)\end{array}$ \\
\hline Age $\geq 75$ & $7(29.2 \%)$ & $1(16.7 \%)$ & $40(22.9 \%)$ & $132(75.4 \%)$ \\
Hypertension & $19(79.2 \%)$ & $5(83.3 \%)$ & $64(36.6 \%)$ & .796 \\
Diabetes & $9(37.5 \%)$ & $5(83.3 \%)$ & $36(20.6 \%)$ & .927 \\
Dyslipidemia & $5(25.0 \%)$ & $0(0 \%)$ & $63(36.0 \%)$ & .087 \\
Smoking & $9(37.5 \%)$ & $3(50.0 \%)$ & $47(26.9 \%)$ & .513 \\
Cardiac disease & $6(25.0 \%)$ & $2(33.3 \%)$ & $143(81.7 \%)$ & .661 \\
Stroke-TIA & $21(87.5 \%)$ & $5(83.3 \%)$ & $84(48.0 \%)$ & .829 \\
Alcohol & $9(37.5 \%)$ & $4(66.7 \%)$ & $58(33.1 \%)$ \\
Previous stroke & $8(33.3 \%)$ & $0(0 \%)$ & .770 \\
\hline
\end{tabular}

Table 3: Event rate and outcomes ${ }^{a}$

\begin{tabular}{|c|c|c|c|c|}
\hline \multirow[b]{2}{*}{ Events and Outcomes } & \multicolumn{2}{|c|}{ Bilateral Group } & \multirow{2}{*}{$\begin{array}{c}\text { Unilateral Group } \\
\text { Control } \\
(n=175)\end{array}$} & \multirow[b]{2}{*}{$P$ Value } \\
\hline & $\begin{array}{l}\text { Simultaneous } \\
\quad(n=24)\end{array}$ & $\begin{array}{l}\text { Staged } \\
(n=6)\end{array}$ & & \\
\hline Hyperperfusion syndrome & 4 & 0 & 5 & .036 \\
\hline Hemodynamic depression & 7 & 3 & 49 & .505 \\
\hline \multicolumn{5}{|l|}{ 6-month event } \\
\hline Minor stroke & $2(2)$ & 0 & $8(6)$ & 1.000 \\
\hline Major stroke & 0 & 0 & $4(4)$ & 1.000 \\
\hline Death & 1 & 0 & $7(2)$ & .550 \\
\hline \multicolumn{5}{|l|}{ 6-month outcome } \\
\hline $\mathrm{mRs} \leq 2$ & 23 & 6 & 158 & .830 \\
\hline$m R s>2$ & 1 & 0 & 17 & \\
\hline Restenosis & $3 / 36(8.3 \%)$ & $0 / 12$ & $9 / 132(6.8 \%)$ & .321 \\
\hline Total deaths & 1 & 0 & 7 & .676 \\
\hline
\end{tabular}

a The number in parentheses in a 6-month event refers to a 1-month event.

$<50$ beats/min) regardless of the need for adjunctive atropine, fluid support, or vasopressor agents, was defined as HD. ${ }^{22}$

All patients underwent independent carotid duplex sonography or CT angiography follow-up as well as evaluation by an independent neurologist using the NIHSS and the mRS. The occurrence of adverse events was evaluated at 30 days and again at 6 months after the procedure.

The clinical outcome measures, including minor/major stroke and death, were defined as follows: minor stroke was defined as a new nondisabling neurologic deficit or as an increase in NIHSS by 3 , but which completely resolved within 30 days. ${ }^{23}$ Major stroke was defined as a new neurologic deficit with an NIHSS score increased by 4 that persisted $>30$ days. The final outcome at 6 months following the procedure was determined by the mRS (good outcome, $\leq 2$; poor outcome, $\geq 3$ ).

All patients, excluding 8 patients who subsequently died and 1 patient with unilateral lesions who was lost to 6-month follow-up, were clinically followed for 6 months. If patients were not followed in an outpatient clinic, an experienced nurse telephoned them $(n=11)$ to evaluate the possibility of any clinically relevant event, and their mRS function outcome was adjusted, including dependency, living situation, mobility, dressing, and toilet functions. ${ }^{23}$ Follow-up imaging for restenosis at 6 months was performed in 24 patients (80\%) with bilateral lesions by using Doppler sonography $(n=22)$ or CT angiography $(n=2)$ and in 132 patients $(75 \%)$ with unilateral lesions by using Doppler sonography $(n=115)$, cerebral conventional angiography $(n=9)$, or CT angiography $(n=8)$. In-stent restenosis was identified by Doppler sonography. In-stent restenosis was defined when the peak systolic velocity was $>300 \mathrm{~cm} / \mathrm{s}^{24}$

\section{Statistical Analysis}

All values are expressed as the mean \pm SD. Bivariate analysis of the demographics and variables to identify potential stroke risk factors, including event rate and outcomes, was performed by using the $\chi^{2}$ test and the Fisher exact test. Variables including the lesion length, preand poststenosis rate, and outcome, including the restenosis rate, were compared by using the Kruskal-Wallis equality-of-populations rank test. All reported probability values were 2 -sided, and a value of $P<.05$ was considered to be statistically significant. Statistical analysis was performed by using SAS software (Version 9.1.3; SAS Institute, Cary, North Carolina).

\section{Results}

The details of the risk factors for CAS in the study patients, including demographics, clinical characteristics, and atherosclerosis risk factors, as well as the detailed analysis of the risk factors for each patient group and appropriate levels of statistical significance, are shown in Table 2. There was no statistically significant difference in the preoperative patient $\geq 75$ years of age or the incidence of hypertension, dyslipidemia, diabetes, smoking, cardiac disease, symptom pattern (stroke versus TIA), alcohol use, or previous stroke in either patient group.

Postprocedural events and follow-up outcome data are shown in Table 3. HPS occurred in 4 of 24 patients $(16.7 \%)$ after SBCAS and in 5 of 175 patients $(2.9 \%)$ in the control group. The comparison of HPS incidence demonstrated a statistical difference among the 3 groups $(P=.036)$. Further comparison showed a higher incidence of occurrence in the 
SBCAS group than in the control group $(P=.014)$. HPS occurred in 4 patients with SBCAS within 1 day $(n=2), 11$ days $(n=1)$, and 3 weeks $(n=1)$ after stent placement, whereas all 5 cases of HPS occurred within 1 day after stent placement in the unilateral CAS group. There were 2 minor strokes in 4 patients with HPS in the SBCAS group, and 1 minor and 2 major stokes in patients with HBS in the unilateral CAS group. ${ }^{25}$

The incidence of HD did not statistically differ in the 2 groups $(P=.505)$ (Table 3$)$. Thirty-two $(54.2 \%)$ of 59 patients with HD had transient change and subsequently recovered, whereas $27(45.8 \%)$ patients received either vasopressor or anticholinergic drugs during the 24 hours following CAS treatment. No patient required transcutaneous or transvenous pacing.

At 30 days following stent placement, there were 2 adverse events, including 2 minor strokes in the SBCAS group, compared with 12 adverse events, including 6 minor strokes, 4 major strokes, and 2 deaths, in the unilateral stent-placement group. The 2 deaths were caused by ipsilateral frontal lobe hematoma in 1, with intraventricular hemorrhage, which occurred 2 days after the stent-placement procedure, perhaps due to hypertensive hemorrhage, which led to death 2 weeks later, and by unidentified etiology in 1 patient.

At 6 months following stent placement, there was 1 death related to renal insufficiency in a patient with SBCAS compared with 2 minor strokes and 5 deaths (ie, iliac artery rupture in 1 patient, myocardial infarction and chronic renal failure in 1 , congestive heart failure and sepsis in 1 , sepsis caused by infective endocarditis in 1, and respiratory insufficiency in 1) in the patients in the unilateral stent-placement group. In 2 patients with chronic renal failure, renal dysfunction already existed before carotid stent placement and was not directly related to the contrast agent administration during the stentplacement procedure.

Restenosis occurred within 6 months following stent placement in 3 patients $(8.3 \%)$ in the SBCAS group and in 9 patients $(6.8 \%)$ in the control group. There was no stroke, death, or restenosis in the 6 patients with staged CAS. Comparison of these follow-up outcomes did not show a statistically significant difference at 30 days or 6 months.

\section{Discussion}

Bilateral carotid stenosis is not uncommon and is encountered in $\leq 51 \%$ of patients undergoing the CAS procedure. ${ }^{9,17,26,27}$ The reported rate of severe bilateral carotid stenosis and/or occlusion varies widely $(3.2 \%-39 \%)^{8,9,17,28}$ and was encountered in $14.6 \%$ of the patients at high surgical risk in our prospective CAS data base. Although bilateral carotid stenosis has been shown to potentially increase the risk for complications during CEA or CAS and has been included as 1 of the high risks for CEA, our data revealed that there was no significant difference in outcome at 30 days or at 6 months between the bilateral and unilateral stent-placement patient groups. ${ }^{1,10,29-31}$

Although the outcome at 6 months did not reveal a significant difference in our study, the incidence of HPS in the SBCAS group was higher than that in the unilateral stent-placement group $(P=.036)$. One characteristic difference we observed in our study was the onset of HPS, because 2 of 4 patients developed it 11 days and 3 weeks, respectively, after
SBCAS in contrast to all 5 patients who developed HPS within 1 day following the stent placement procedure in the unilateral stent placement group. Therefore, our data suggested that careful management of BP is required for almost 1 month after BSCAS to avoid HPS, though the incidence was relatively low. ${ }^{1,21,32}$

Although staged CAS, because it may decrease the occurrence of HPS and HD, is more acceptable and therefore more frequently performed, there are obvious disadvantages to a staged intervention, including the higher medical cost and inconvenience to the patient. It may also potentially cause the delay of life-saving treatment such as open-heart surgery or even cause another cerebral infarction. ${ }^{33}$ Furthermore, the most reasonable timing for the sequential stent-placement procedure when both carotid arteries are involved has still not been determined. ${ }^{34}$ Although no criteria have been established to indicate the optimal timing of the subsequent stent placement procedure, a 1-month delay for contralateral revascularization therapy has prevailed during the recent past. ${ }^{17}$ In our study, the median time for subsequent stent placement in the 6 patients was 33 days.

Another theoretic risk of SBCAS is HD with persistent severe bradycardia and hypotension caused by activation of the bilateral carotid sinus reflex. However, the incidence of HD in patients with SBCAS is not higher than that in those with staged bilateral CAS or unilateral CAS. Finally, the total incidence of HD in our series was not higher than that in reported studies. ${ }^{18,22,35-37}$ All of the patients with HD in our study recovered spontaneously or after infusion of normal saline and/or volume expanders or occasionally with the use of vasopressors and/or anticholinergics. HD during CAS is relatively common and benign, without an increase of perioperative risks. Two limitations of this study are the retrospective nature of the analysis as well as the limited number of patients. A randomized prospective study could further clarify the outcome difference between staged and simultaneous stent placement for bilateral severe carotid stenosis.

\section{Conclusions}

The outcome after SBCAS revealed no significant differences compared with those of unilateral carotid stent placement at 30 days and 6 months following the procedure. More HPS occurred with SBCAS, which, in most cases, is preventable by careful monitoring of patient BP status even after discharge, because HPS can occur $\leq 30$ days following the stent-placement procedure, especially after SBCAS. HD after SBCAS is usually a transient and benign event, which does not increase the procedural risks of CAS.

\section{Acknowledgments}

We acknowledge the assistance of Sun Moon Whang, BS, and Eun Hye Kim, RN, in the patient data collection, as well as that of Yun Gyeong Jeong in the manuscript preparation. We thank Bonnie Hami, MA (United States) for her English editorial assistance.

\section{References}

1. Bates ER, Babb JD, Casey DE Jr, et al. ACCF/SCAI/SVMB/SIR/ASITN 2007 clinical expert consensus document on carotid stenting: a report of the American College of Cardiology Foundation Task Force on Clinical Expert Consen- 
sus Documents (ACCF/SCAI/SVMB/SIR/ASITN Clinical Expert Consensus Document Committee on Carotid Stenting). J Am Coll Cardiol 2007;49:126-70

2. Balzer JO. How to introduce carotid angioplasty without compromising patient safety. Eur J Vasc Endovasc Surg 2008;36:138-44. Epub 2008 Jun 24

3. McKinsey JF. Symptomatic carotid stenosis: endarterectomy, stenting, or best medical management? Semin Vasc Surg 2008;21:108-14

4. Mozes G, Sullivan TM, Torres-Russotto DR, et al. Carotid endarterectomy in SAPPHIRE-eligible high-risk patients: implications for selecting patients for carotid angioplasty and stenting. J Vasc Surg 2004;39:958-65, discussion 65-66

5. Levy EI, Mocco J, Samuelson RM, et al. Optimal treatment of carotid artery disease. J Am Coll Cardiol 2008;51:979-85

6. Mathur A, Roubin GS, Yadav JS, et al. Combined coronary and bilateral carotid stenting: a case report. Cathet Cardiovasc Diagn 1997;40:202-06

7. Bali HK, Bhargava M, Bhatta YK, et al. Single stage bilateral common carotid artery stenting in a patient of Takayasu arteritis. Neurol India 2001;49:87-90

8. Henry M, Amor M, Klonaris C, et al. Angioplasty and stenting of the extracranial carotid arteries. Tex Heart Inst J 2000;27:150-58

9. Mathur A, Roubin GS, Iyer SS, et al. Predictors of stroke complicating carotid artery stenting. Circulation 1998;97:1239-45

10. Henry M, Gopalakrishnan L, Rajagopal S, et al. Bilateral carotid angioplasty and stenting. Catheter Cardiovasc Interv 2005;64:275-82

11. Lesley WS, Lazo A, Kazmierczak CD, et al. Simultaneous bilateral carotid stenting for postendarterectomy restenosis. Catheter Cardiovasc Interv 2003;58: $147-50$

12. Chen MS, Bhatt DL, Mukherjee D, et al. Feasibility of simultaneous bilateral carotid artery stenting. Catheter Cardiovasc Interv 2004;61:437-42

13. Al-Mubarak N, Roubin GS, Vitek JJ, et al. Simultaneous bilateral carotid stenting for restenosis after endarterectomy. Cathet Cardiovasc Diagn 1998;45:11-15

14. Wang YH, Hsieh HJ, Lee CW, et al. Simultaneous bilateral carotid stenting in one session in high-risk patients. I Neuroimaging 2008;18:252-55

15. Halse O, Clifton A, Cloud GC. Endovascular intervention for symptomatic bilateral carotid artery stenosis in an octogenarian. Age Ageing 2007;36: 102-04

16. Pyun HW, Suh DC, Kim JK, et al. Concomitant multiple revascularizations in supra-aortic arteries: short-term results in $\mathbf{5 0}$ patients. AJNR Am J Neuroradiol 2007;28:1895-901

17. Diehm N, Katzen BT, Iyer SS, et al. Staged bilateral carotid stenting, an effective strategy in high-risk patients: insights from a prospective multicenter trial. J Vasc Surg 2008; 47:1227-34

18. Qureshi AI, Luft AR, Sharma M, et al. Frequency and determinants of postprocedural hemodynamic instability after carotid angioplasty and stenting. Stroke 1999;30:2086-93

19. Gasecki AP, Eliasziw M, Ferguson GG, et al. Long-term prognosis and effect of endarterectomy in patients with symptomatic severe carotid stenosis and contralateral carotid stenosis or occlusion: results from NASCET-North American Symptomatic Carotid Endarterectomy Trial (NASCET) Group. J Neurosurg 1995;83:778-82

20. Lee DK, Kim JS, Kwon SU, et al. Lesion patterns and stroke mechanism in atherosclerotic middle cerebral artery disease: early diffusion-weighted imaging study. Stroke 2005;36:2583-88

21. Abou-Chebl A, Yadav JS, Reginelli JP, et al. Intracranial hemorrhage and hyperperfusion syndrome following carotid artery stenting: risk factors, prevention, and treatment. J Am Coll Cardiol 2004;43:1596-601

22. Cieri E, De Rango P, Maccaroni MR, et al. Is haemodynamic depression during carotid stenting a predictor of peri-procedural complications? Eur J Vasc Endovasc Surg 2008;35:399-404

23. Goldstein LB, Samsa GP. Reliability of the National Institutes of Health Stroke Scale: extension to non-neurologists in the context of a clinical trial. Stroke 1997;28:307-10

24. Setacci C, Chisci E, Setacci F, et al. Grading carotid intrastent restenosis: a 6-year follow-up study. Stroke 2008;39:1189-96

25. Cho AH, Suh DC, Kim GE, et al. MRI evidence of reperfusion injury associated with neurological deficits after carotid revascularization procedures. Eur J Neurol 2009;16:1066-69. Epub 2009 May 22

26. Roubin GS, New G, Iyer SS, et al. Immediate and late clinical outcomes of carotid artery stenting in patients with symptomatic and asymptomatic carotid artery stenosis: a 5-year prospective analysis. Circulation 2001;103: 532-37

27. White CJ, Iyer SS, Hopkins LN, et al. Carotid stenting with distal protection in high surgical risk patients: the BEACH trial 30 day results. Catheter Cardiovas Interv 2006;67:503-12

28. Lavoie P, Rutledge J, Dawoud MA, et al. Predictors and timing of hypotension and bradycardia after carotid artery stenting. AJNR Am J Neuroradiol 2008;29:1942-47

29. Darling RC 3rd, Kubaska S, Shah DM, et al. Bilateral carotid endarterectomy during the same hospital admission. Cardiovasc Surg 1996;4:759-62

30. Farsak B, Oc M, Boke E. Simultaneous bilateral carotid endarterectomy: our first experience. Ann Thorac Cardiovasc Surg 2001;7:292-96

31. Anzola GP, Limoni P, Cavrini G. Predictors of carotid clamping intolerance during endarterectomy that would be wise to apply to stenting procedures. Cerebrovasc Dis 2008;26:494-501

32. Abou-Chebl A, Reginelli J, Bajzer CT, et al. Intensive treatment of hypertension decreases the risk of hyperperfusion and intracerebral hemorrhage following carotid artery stenting. Catheter Cardiovasc Interv 2007;69:690-96

33. Groschel K, Knauth M, Ernemann U, et al. Early treatment after a symptomatic event is not associated with an increased risk of stroke in patients undergoing carotid stenting. Eur J Neurol 2008;15:2-5

34. Naylor AR. Delay may reduce procedural risk, but at what price to the patient? Eur J Vasc Endovasc Surg 2008;35:383-91

35. Leisch F, Kerschner K, Hofmann R, et al. Carotid sinus reactions during carotid artery stenting: predictors, incidence, and influence on clinical outcome. Catheter Cardiovasc Interv 2003;58:516-23

36. Taha MM, Toma N, Sakaida H, et al. Periprocedural hemodynamic instability with carotid angioplasty and stenting. Surg Neurol 2008;70:279-85

37. Trocciola SM, Chaer RA, Lin SC, et al. Analysis of parameters associated with hypotension requiring vasopressor support after carotid angioplasty and stenting. J Vasc Surg 2006;43:714-20 